TOKYO J. MATH.

VOL. 36, NO. 2, 2013

\title{
Vector-valued Maximal Inequalities on Weighted Orlicz-Morrey Spaces
}

\author{
Kwok-Pun HO \\ The Hong Kong Institute of Education \\ (Communicated by H. Kikuchi)
}

\begin{abstract}
The Fefferman-Stein vector-valued maximal inequalities are established for weighted OrliczMorrey spaces. As an application of these inequalities, a framework of weighted Triebel-Lizorkin-Orlicz-Morrey spaces is proposed.
\end{abstract}

\section{Introduction}

The main theme of this paper is the Fefferman-Stein vector-valued maximal inequalities on weighted Orlicz-Morrey spaces. The study of vector-valued maximal inequalities was initiated by Fefferman and Stein in [5].

After Fefferman and Stein proved vector-valued maximal inequalities in [5], a passage to a number of important function spaces in harmonic analysis is done by many people.

In [1], the vector-valued maximal inequalities on the weighted Lebesgue spaces were obtained. The vector-valued maximal inequalities on Orlicz spaces, in term of modular and norm, were established in [12, Theorem 1.3.3] and [12, Theorem 1.3.5], respectively. In [29], the vector-valued maximal inequalities was generalized to Morrey spaces.

In fact, the result in [29] provides an access to solve a conjecture proposed by Mazzucato [15] for the study of Morrey type Triebel-Lizorkin spaces [26, 30]. It further inspired the study in [7] which showed that, roughly speaking, the validity of the vector-valued maximal inequalities on a Banach function space $X$ can guarantee that the Triebel-Lizorkin type space on $X$ is well defined and possesses atomic and molecular decompositions [7, 8]. Furthermore, the extension of the vector-valued maximal inequalities to rearrangement-invariant (r.-i.) quasi-Banach spaces and the corresponding Morrey type space were obtained in [7].

In this paper, we further generalize the vector-valued maximal inequalities to weighted Orlicz-Morrey spaces. Notice that there is another family of Orlicz-Morrey spaces studied in [7, Section 5] but they are different from the ones given here. The weighted Orlicz-Morrey

Received August 17, 2012; revised September 10, 2012

2000 Mathematics Subject Classification: 42B25, 42B35, 46A80, 46E30, 46E40

Key words and phrases: Vector-valued maximal inequality, weight function, Orlicz spaces, Morrey spaces, TriebelLizorkin-Orlicz-Morrey spaces 
space $M_{\Phi, \omega}^{u}$ given in this paper is defined via the modular while the ones used in [7] $\mathcal{M}_{\Phi}^{v}$ can be viewed as the Morrey space generated by the norm. The reader is referred to Definitions 3.1 and 4.1 to see the difference.

In this paper, for comparison and completeness, we also provide the vector-valued maximal inequalities on $\mathcal{M}_{\Phi}^{v}$. Furthermore, the reader can also compare the conditions, (3.2) and (4.7), imposed on the weight functions $u(x, r)$ and $v(x, r)$ for $M_{\Phi, \omega}^{u}$ and $\mathcal{M}_{\Phi}^{v}$, respectively, to see the dissimilitude between $M_{\Phi, \omega}^{u}$ and $\mathcal{M}_{\Phi}^{v}$.

This paper is organized as follows. Section 2 provides the definitions and some preliminaries on weight functions and Orlicz spaces. The weighted Orlicz-Morrey spaces are introduced in Section 3. The main result is established in Section 4. An application of the main result to weighted Triebel-Lizorkin-Orlicz-Morrey spaces is also given at the end of Section 4.

\section{Definitions and preliminaries}

Let $B(z, r)=\left\{x \in \mathbf{R}^{n}:|x-z|<r\right\}$ denote the open ball with center $z \in \mathbf{R}^{n}$ and radius $r>0$. Let $\mathcal{B}=\left\{B(z, r): z \in \mathbf{R}^{n}, r>0\right\}$. Let $\mathcal{P}$ denote the class of polynomials on $\mathbf{R}^{n}$.

We first recall the definition of the well-known Muckenhoupt weight functions.

Definition 2.1. For $1<p<\infty$, a locally integrable function $\omega: \mathbf{R}^{n} \rightarrow[0, \infty)$ is said to be an $A_{p}$ weight if

$$
\sup _{B \in \mathcal{B}}\left(\frac{1}{|B|} \int_{B} \omega(x) d x\right)\left(\frac{1}{|B|} \int_{B} \omega(x)^{-\frac{p^{\prime}}{p}} d x\right)^{\frac{p}{p^{\prime}}}<\infty
$$

where $p^{\prime}=\frac{p}{p-1}$. A locally integrable function $\omega: \mathbf{R}^{n} \rightarrow[0, \infty)$ is said to be an $A_{1}$ weight if

$$
\frac{1}{|B|} \int_{B} \omega(y) d y \leq C \omega(x), \quad \text { a.e. } x \in B
$$

for some constant $C>0$ which is independent of the balls $B$. We define $A_{\infty}=\cup_{p \geq 1} A_{p}$.

A function $\Phi:[0,+\infty] \rightarrow[0,+\infty]$ is a Young function if there exists an increasing and left-continuous function $\phi$ satisfying $\phi(0)=0$ and that $\phi$ is neither identically zero nor identically infinite such that

$$
\Phi(s)=\int_{0}^{s} \phi(u) d u, \quad s \geq 0 .
$$

A Young function $\Phi$ is said to satisfy the $\triangle_{2}$-condition if there exists a constant $K>0$ such that

$$
\Phi(2 t) \leq K \Phi(t), \quad t>0 .
$$

We write $\Phi \in \triangle_{2}$ if it satisfies the $\triangle_{2}$-condition. 
Let $\Phi$ be a Young function associated with $\phi$. Let

$$
\psi(v)=\inf \{u \geq 0: \phi(u) \geq v\}, \quad 0 \leq v \leq \infty .
$$

The function $\Psi$ defined by

$$
\Psi(t)=\int_{0}^{t} \psi(v) d v, \quad 0 \leq t \leq \infty
$$

is called the conjugate (complementary) function of $\Phi$ [2, Chapter 4, Definition 8.11].

We write $\Phi \in \nabla_{2}$ if there exists a constant $K>0$ such that

$$
\Psi(2 t) \leq K \Psi(t)
$$

where $\Psi$ denotes the conjugate function of $\Phi$.

For any $\sigma$-finite measure $\mu$ on $\mathbf{R}^{n}$ defined on Lebesgue measurable sets, the Orlicz space with respect to $\mu, L_{\Phi, \mu}$, consists of all Lebesgue measurable functions $f$ satisfying

$$
\|f\|_{L_{\Phi, \mu}}=\inf \left\{\lambda>0: \int_{\mathbf{R}^{n}} \Phi(|f| / \lambda) d \mu \leq 1\right\}<\infty .
$$

Particularly, the weighted Orlicz space $L_{\Phi, \omega}$ associated with the Young function $\Phi$ and $\omega \in A_{\infty}$ is the collection of all Lebesgue measurable functions $f$ such that

$$
\|f\|_{L_{\Phi, \omega}}=\inf \left\{\lambda>0: \int_{\mathbf{R}^{n}} \Phi(|f(x)| / \lambda) \omega(x) d x \leq 1\right\}<\infty .
$$

For any $\sigma$-finite measure $\mu$ on $\mathbf{R}^{n}$, we find that

$$
\int_{\mathbf{R}^{n}} \Phi(|f(x)|) d \mu=\int_{0}^{\infty} \Phi^{\prime}(\lambda) \mu\left(\left\{t: f_{\mu}^{*}(t)>\lambda\right\}\right) d \lambda=\int_{0}^{\infty} \Phi\left(f_{\mu}^{*}(t)\right) d t
$$

where $f_{\mu}^{*}$ is the non-increasing rearrangement of $f$ with respect to $\mu$. Obviously, $L_{\Phi, \omega}$ is a rearrangement-invariant Banach function space with respect to the measure $\omega(x) d x$. In addition, if we denote the collection of all Lebesgue measurable functions $g$ on $[0, \infty]$ satisfying

$$
\|g\|_{L_{\Phi}(0, \infty)}=\inf \left\{\lambda>0: \int_{0}^{\infty} \Phi(|g(t)| / \lambda) d t \leq 1\right\}<\infty
$$

by $L_{\Phi}(0, \infty)$, the function space $L_{\Phi}(0, \infty)$ is the Luxemburg representation of $L_{\Phi, \mu}$, see [2, Chapter 2, Theorem 4.10].

Moreover, any Lebesgue measurable set $E$, we have $\left\|\chi_{E}\right\|_{L_{\Phi, \mu}}=\frac{1}{\Phi^{-1}(1 / \mu(E))}$ where $\Phi^{-1}$ denotes the right-continuous inverse of $\Phi$ given by

$$
\Phi^{-1}(t)=\sup _{s \geq 0} \Phi(s) \leq t, \quad 0 \leq t<\infty
$$


Furthermore, we have the Hölder inequality for Orlicz spaces. That is,

$$
\left|\int_{\mathbf{R}^{n}} f(x) g(x) d \mu(x)\right| \leq C\|f\|_{L_{\Phi, \mu}}\|g\|_{L_{\Psi, \mu}}
$$

for some $C>0$ where $L_{\Psi, \mu}$ denote the Orlicz space generated by $\Psi$, see [23, Section 6.7.14.8].

We recall an important pair of indices used for Young function. For any Young function $\Phi$, write

$$
h_{\Phi}(t)=\sup _{s>0} \frac{\Phi(s t)}{\Phi(s)}, \quad t>0 .
$$

The lower and upper dilation indices of $\Phi$ are defined by

$$
i_{\Phi}=\lim _{t \rightarrow 0^{+}} \frac{\log h_{\Phi}(t)}{\log t} \text { and } I_{\Phi}=\lim _{t \rightarrow \infty} \frac{\log h_{\Phi}(t)}{\log t},
$$

respectively.

For any rearrangement-invariant Banach function space $X$, we denote its lower and upper Boyd indices by $p_{X}$ and $q_{X}$, respectively, see [14, Volume II, Definition 2.b.1] and [2, Chapter 5.10, Definitions 5.10 and 5.12]. Notice that the Boyd indices given in [2] are the reciprocal of the ones given in [14].

As the Boyd indices of rearrangement-invariant Banach function space $X$ are defined via the boundedness of the dilation operator on the Luxemburg representation of $X$ (see [2, Chapter 5.10, Definitions 5.10 and 5.12]), the Boyd indices of $L_{\Phi, \mu}$ and $L_{\Phi}(0, \infty)$ are equal by definition. Furthermore, the Boyd indices of $L_{\Phi}(0, \infty)$ are equal to the dilation indices of $\Phi$, respectively (see [14, Volume II, Proposition 2.b.5]). Therefore, the lower and upper Boyd indices of $L_{\Phi, \mu}$ are the same as the lower and upper dilation indices of $\Phi$, respectively. More precisely,

$$
p_{L_{\Phi, \mu}}=i_{\Phi}, \quad \text { and } \quad q_{L_{\Phi, \mu}}=I_{\Phi} .
$$

In addition, $\Phi \in \triangle_{2} \cap \nabla_{2}$ if and only if $1<p_{L_{\Phi}} \leq q_{L_{\Phi}}<\infty$.

The proof of our main result requires some geometric properties of Banach function spaces. We first recall the notions of $p$-convex and $p^{\text {th }}$-power of Banach function spaces from [22, Section 2.2].

Definition 2.2. Let $0 \leq p<\infty$. A Banach function space $X$ is said to be $p$-convex if its norm $\|\cdot\|_{X}$ satisfies

$$
\left\|\left(\sum_{i=1}^{n}\left|v_{i}\right|^{p}\right)^{\frac{1}{p}}\right\|_{X} \leq M\left(\sum_{i=1}^{n}\left\|v_{i}\right\|_{X}^{p}\right)^{\frac{1}{p}}
$$

for any finite set $\left\{v_{i}\right\}_{i=1}^{n} \subset X$, where $M$ is a constant independent of $\left\{v_{i}\right\}_{i=1}^{n}$. 
Definition 2.3. Let $0<p<\infty$. Let $X$ be a $p$-convex Banach function space. The $p^{t h}$-power of $X$ consists of all $f$ so that $|f|^{1 / p} \in X$. We denote the $p^{t h}$-power of $X$ by $X_{[p]}$ and it is endowed with the quasi-norm

$$
\|f\|_{X_{[p]}}=\left\||f|^{\frac{1}{p}}\right\|_{X}^{p} .
$$

We find that whenever $X$ is $p$-convex, then $X_{[p]}$ is a Banach function space (see [22, Proposition 2.23]). That is, $\|\cdot\|_{X_{[p]}}$ is a norm.

LEMMA 2.1. Let $\Phi$ be a Young function and $\mu$ be a $\sigma$-finite measure. Then, for any $r<i_{\Phi}, L_{\Phi, \mu}$ is an $r$-convex Banach function space.

Proof. According to [14, Volume II, Proposition 2.b.5], we have

$$
p_{L_{\Phi, \mu}}=\sup \left\{p: L_{\Phi, \mu} \text { satisfies an upper } p \text {-estimate }\right\} .
$$

Furthermore, in view of [14, Volume II, Theorem 1.f.7], for any $r<p_{L_{\Phi, \mu}}, L_{\Phi, \mu}$ is $r$-convex. Our promised result follows from the fact that $i_{\Phi}=p_{L_{\Phi, \mu}}$.

For any Banach function space $X$, denote the associate space of $X$ by $X^{\prime}$. For the definition of associate space, the reader is referred to [2, Chapter 1, Definitions 2.1 and 2.3]. From the above lemma, we find that the $r^{t h}$-power of $L_{\Phi, \mu}$ is a rearrangement-invariant (r.-i.) Banach function space provided that $r<i_{\Phi}$.

As $L_{\Phi, \omega}$ is a r.-i. Banach function space with respect to $\mu$, by using [2, Chapter 5, Theorem 5.2], for any Lebesgue measurable set $E$, we have

$$
\left\|\chi_{E}\right\|_{\left(L_{\Phi, \mu}\right)_{[r]}^{\prime}}=\frac{\mu(E)}{\left\|\chi_{E}\right\|_{\left(L_{\Phi, \mu}\right)_{[r]}}}=\frac{\mu(E)}{\left\|\chi_{E}\right\|_{L_{\Phi, \mu}}^{r}} .
$$

\section{Weighted Orlicz-Morrey spaces}

Definition 3.1. Let $\Phi$ be a Young function, $\omega \in A_{\infty}$ and $u: \mathbf{R}^{n} \times(0, \infty) \rightarrow(0, \infty)$ be a Lebesgue measurable function. The weighted Orlicz-Morrey space $M_{\Phi, \omega}^{u}$ consists of all Lebesgue measurable functions $f$ such that

$$
\|f\|_{M_{\Phi, \omega}^{u}}=\sup _{B \in \mathcal{B}}\|f\|_{(\Phi, u, B, \omega)}<\infty
$$

where

$$
\|f\|_{(\Phi, u, B, \omega)}=\inf \left\{\lambda>0: \frac{1}{u(x, r)} \int_{B(x, r)} \Phi(|f(y)| / \lambda) \omega(y) d y \leq 1\right\}<\infty
$$

and $B=B(x, r) \in \mathcal{B}$.

If $\omega \equiv 1$ and $u(x, r)=r^{n} \phi\left(r^{n}\right)$ where $\phi(t)$ is almost decreasing and $\phi(t) t$ is almost increasing, then the Orlicz-Morrey spaces given in Definition 3.1 reduce to the ones defined and studied in [20]. 
The above definition of Orlicz-Morrey spaces can be further generalized. For instance, if we replace the weighted Lebesgue measure $\omega(x) d x$ by a Radon measure $\mu$ satisfying some growth conditions, then we obtain the Orlicz-Morrey spaces for $\mu$-measurable function in [27]. In addition, another direction for further generalizing our study is the Morrey spaces for non-doubling measures, see [25].

Furthermore, the readers are also referred to [28] for another family of Orlicz-Morrey spaces where a study of the mapping properties of the fractional operators on this family of Orlicz-Morrey spaces is given.

Form the preceding definition, we find that $M_{\Phi, \omega}^{u}$ is defined via the family of modulars

$$
\rho_{B}(f)=\frac{1}{u(B)} \int_{B} \Phi(|f(y)|) \omega(y) d y .
$$

The reader may consult $[17,21]$ for the study of modular and its relation with function spaces.

When $\omega \equiv 1$, we write $M_{\Phi, \omega}^{u}$ as $M_{\Phi}^{u}$. Moreover, We write $M_{p}^{u}$ to denote the unweighted Orlicz-Morrey space associated with $\Phi(t)=t^{p}, 1 \leq p<\infty$ and $\omega \equiv 1$. Note that if we define $\mu$ to be the restriction of the measure $\frac{1}{u(B)} \omega(x) d x$ on $B$, then we find that the norms $\|\cdot\|_{L_{\Phi, \mu}}$ and $\|\cdot\|_{(\Phi, u, B, \omega)}$ are identical.

We now identify the condition imposed on $\Phi, \omega$ and $u$ so that the vector-valued maximal inequalities are valid for weighted Orlicz-Morrey spaces.

Definition 3.2. Let $\Phi$ be a Young function and $\omega \in A_{\infty}$. A Lebesgue measurable function $u: \mathbf{R}^{n} \times(0, \infty) \rightarrow(0, \infty)$ is said to be an Orlicz-Morrey weight function for $L_{\Phi, \omega}$ if for any $r>0$ and $x \in \mathbf{R}^{n}$.

$$
\sum_{j=0}^{\infty} \Phi^{-1}\left(\frac{u\left(x, 2^{j} r\right)}{\omega\left(B\left(x, 2^{j} r\right)\right)}\right)<C \Phi^{-1}\left(\frac{u(x, r)}{\omega(B(x, r))}\right)
$$

for some $C>0$ independent of $r>0$ and $x \in \mathbf{R}^{n}$.

We also write $u(B)=u(x, r)$ when $B=B(x, r) \in \mathcal{B}$.

When $\Phi(t)=t^{p}$, the above condition reduces to

$$
\sum_{j=0}^{\infty}\left(\frac{u\left(x, 2^{j} r\right)}{\omega\left(B\left(x, 2^{j} r\right)\right)}\right)^{\frac{1}{p}}<C\left(\frac{u(x, r)}{\omega(B(x, r))}\right)^{\frac{1}{p}}
$$

which is precisely, with some simple modifications on notations, the condition used in [11, Theorem 2.1] to establish the vector-valued maximal inequality for weighted Morrey spaces.

In case $u(x, t)$ satisfies the (uniform) doubling condition

$$
C^{-1} u(x, s) \leq u(x, t) \leq C u(x, s), \quad 0<s \leq t \leq 2 s,
$$


then (3.2) can be rewritten in the integral form

$$
\int_{r}^{\infty} \Phi^{-1}\left(\frac{u(x, t)}{\omega(B(x, t))}\right) \frac{d t}{t} \leq C \Phi^{-1}\left(\frac{u(x, r)}{\omega(B(x, t))}\right)
$$

because $\omega \in A_{\infty}$ ensures that $\omega$ satisfies the doubling property.

Some simple conditions imposed on $\Phi, u$ and $\omega$ can assure the validity of (3.2). For instance, if there exists a $0<\theta<1$ such that

$$
\frac{\omega(B(x, r))}{\omega(B(x, 2 r))} \leq \theta \frac{u(x, r)}{u(x, 2 r)}
$$

for all $x \in \mathbf{R}^{n}$ and $r>0$, then (3.2) is valid.

In fact, according to [2, Chapter 4 , Theorem 8.18], we have

$$
\frac{1}{q_{L_{\Phi, \omega}}}=\lim _{t \rightarrow 0^{+}} \frac{\log g(t)}{\log t}, \quad \text { where } \quad g(t)=\limsup _{s \rightarrow \infty} \frac{\Phi^{-1}(s)}{\Phi^{-1}(s / t)} .
$$

Therefore, fix $\beta \in\left(\frac{1}{q_{L_{\Phi, \omega}}}, \infty\right)$, we find that

$$
\Phi^{-1}(s)<C t^{\beta} \Phi^{-1}(s / t)
$$

for some $C>0$ when $t$ is sufficiently small enough and $0<s<\infty$.

Consequently, inequality (3.4) yields

$$
\frac{u\left(x, 2^{j} r\right)}{\omega\left(B\left(x, 2^{j} r\right)\right)}<\theta^{j} \frac{u(x, r)}{\omega(B(x, r))}
$$

and (3.2) follows from (3.5) and (3.6).

In particular, when $\omega \in A_{\infty}$ and $u(x, r)=\omega(B(x, r))^{\lambda}, 0<\lambda<1$. Then (3.2) is valid (see [6, Theorem 9.3.3 (d)]).

The above analysis also gives us an insight to obtain the following fundamental property for $M_{\Phi, \omega}^{u}$.

Proposition 3.1. Let $\Phi$ be a Young function, $\omega \in A_{\infty}$ and $u: \mathbf{R}^{n} \times(0, \infty) \rightarrow$ $(0, \infty)$ satisfies (3.3), $u(x, r)>C$ when $r>1$ for some $C>0$ and

$$
\frac{u(x, 2 r)}{u(x, r)} \leq\left(\frac{\omega(B(x, 2 r))}{\omega(B(x, r))}\right)^{\lambda}
$$

for some $0<\lambda<1$. Then $\chi_{B} \in M_{\Phi, \omega}^{u}$ for any $B \in \mathcal{B}$.

Proof. Let $B=B(x, r) \in \mathcal{B}$. For any $z \in \mathbf{R}^{n}$ and $j \in \mathbf{Z}$, define $B_{j}=B\left(z, 2^{j}\right)$. When $j<0, B\left(z, 2^{j}\right) \cap B(x, r)=\emptyset$ if $|x-z|>r+2$. In view of (3.7), we obtain

$$
\frac{u\left(B_{0}\right)\left(\frac{\omega\left(B_{j}\right)}{\omega\left(B_{0}\right)}\right)^{\lambda}}{\omega\left(B_{j}\right)} \leq \frac{u\left(B_{0}\right)\left(\frac{\omega\left(B_{j}\right)}{\omega\left(B_{0}\right)}\right)^{\lambda}}{\omega\left(B_{j} \cap B\right)} \leq \frac{u\left(B_{j}\right)}{\omega\left(B \cap B_{j}\right)} .
$$


Therefore,

$$
\left\|\chi_{B}\right\|_{\left(\Phi, u, B_{j}, \omega\right)}=\frac{1}{\Phi^{-1}\left(\frac{u\left(B_{j}\right)}{\omega\left(B \cap B_{j}\right)}\right)} \leq \frac{1}{\Phi^{-1}\left(\frac{u\left(B_{0}\right)}{\omega\left(B_{0}\right)}\left(\frac{\omega\left(B_{j}\right)}{\omega\left(B_{0}\right)}\right)^{\lambda-1}\right)} \leq \frac{1}{\Phi^{-1}\left(\frac{u\left(B_{0}\right)}{\omega\left(B_{0}\right)}\right)}
$$

where we have the last inequality because $\lambda<1$ and $\omega\left(B_{j}\right) \leq \omega\left(B_{0}\right)$.

Moreover, as $u\left(B_{0}\right)>C$ and $B_{0} \subseteq B(x, r+2)$, we find that

$$
\frac{C}{\omega(B(x, r+2))} \leq \frac{C}{\omega\left(B_{0}\right)} \leq \frac{u\left(B_{0}\right)}{\omega\left(B_{0}\right)} \text {. }
$$

Hence,

$$
\left\|\chi_{B}\right\|_{\left(\Phi, u, B_{j}, \omega\right)} \leq \frac{1}{\Phi^{-1}\left(\frac{C}{\omega(B(x, r+2))}\right)} .
$$

When $j>0$, since $u\left(B_{j}\right)>C$, we have

$$
\left\|\chi_{B}\right\|_{\left(\Phi, u, B_{j}, \omega\right)}=\frac{1}{\Phi^{-1}\left(\frac{u\left(B_{j}\right)}{\omega\left(B \cap B_{j}\right)}\right)} \leq \frac{1}{\Phi^{-1}\left(\frac{C}{\omega(B)}\right)} .
$$

Since $u$ satisfies (3.3), we have $\sup _{D \in \mathcal{B}}\left\|\chi_{B}\right\|_{(\Phi, u, D, \omega)}<\infty$ and conclude that $\chi_{B} \in$ $M_{\Phi, \omega}^{u}$.

Let 1 denote the constant function with value one for every $x \in \mathbf{R}^{n}$. We have

$$
\|\mathbf{1}\|_{(\Phi, u, B, \omega)}=\frac{1}{\Phi^{-1}\left(\frac{u(x, r)}{\omega(B)}\right)}, \quad \text { where } \quad B=B(x, r) .
$$

In addition, we find that Condition (3.2) can be rewritten as

$$
\sum_{j=0}^{\infty}\|\mathbf{1}\|_{\left(\Phi, u, B\left(x, 2^{j} r\right), \omega\right)}^{-1} \leq C\|\mathbf{1}\|_{(\Phi, u, B(x, r), \omega)}^{-1} .
$$

\section{Vector-valued maximal inequalities}

Let us denote the Hardy-Littlewood maximal operator by M. For any family of locally integrable functions $f=\left\{f_{i}\right\}_{i=1}^{\infty}$, write

$$
\mathbf{M} f=\left\{\mathbf{M} f_{i}\right\}_{i=1}^{\infty} .
$$

Before the presentation of the main result, we recall a crucial inequality to establish the vector-valued maximal inequalities in weighted Orlicz-Morrey spaces, the weighted vectorvalued modular inequality for Young function. 
Proposition 4.1. Let $1<q<\infty, \Phi \in \triangle_{2} \cap \nabla_{2}$ be a Young function, $\omega \in A_{i_{\Phi}}$. Then, for any family of locally integrable functions $f=\left\{f_{i}\right\}_{i=1}^{\infty}$, we have

$$
\int_{\mathbf{R}^{n}} \Phi\left(\|\mathbf{M} f(x)\|_{l^{q}}\right) \omega(x) d x \leq C \int_{\mathbf{R}^{n}} \Phi\left(\|f(x)\|_{l^{q}}\right) \omega(x) d x
$$

for some $C>0$ independent of $f$.

The reader is referred to [3, Chapter 4] for the proof of the above result. The unweighted case of Proposition 4.1 was obtained in [12, Theorem 1.3.3].

We are now ready to state and prove our main result.

THEOREM 4.2. Let $1<q<\infty, \Phi \in \triangle_{2} \cap \nabla_{2}$ be a Young function, $\omega \in A_{i_{\Phi}}$ and $u$ be an Orlicz-Morrey weight function for $L_{\Phi, \omega}$. Then, there exists a constant $C>0$ such that for any family of locally integrable functions $f=\left\{f_{i}\right\}_{i=1}^{\infty}$, we have

$$
\|\| \mathbf{M} f\left\|_{l^{q}}\right\|_{M_{\Phi, \omega}^{u}} \leq C\|\| f\left\|_{l^{q}}\right\|_{M_{\Phi, \omega}^{u}} .
$$

Proof. Without loss of generality, we let \|\|$f\left\|_{l^{q}}\right\|_{M_{\Phi, \omega}^{u}}=1$. That is, for any $B(x, R) \in \mathcal{B}$, we have

$$
\int_{B(x, R)} \Phi\left(\|f(y)\|_{l q}\right) \omega(y) d y \leq u(x, R)
$$

For any $z \in \mathbf{R}^{n}$ and $r>0$, write $B_{j}=B\left(z, 2^{j} r\right)$ and $f_{i}=f_{i}^{0}+\sum_{j=1}^{\infty} f_{i}^{j}$, where $f_{i}^{0}=$ $\chi_{B(z, 2 r)} f_{i}$ and $f_{i}^{j}=\chi_{B_{j+1} \backslash B_{j}} f_{i}, j \in \mathbf{N}$. In view of $\omega \in A_{i_{\Phi}}$, by using Proposition 4.1 to $f^{0}=\left\{f_{i}^{0}\right\}_{i \in \mathbf{Z}}$, we obtain

$$
\int_{\mathbf{R}^{n}} \Phi\left(\left\|\mathbf{M} f^{0}(x)\right\|_{l^{q}}\right) \omega(x) d x \leq C \int_{\mathbf{R}^{n}} \Phi\left(\left\|f^{0}(x)\right\|_{l^{q}}\right) \omega(x) d x
$$

for some $C>0$. As supp $f_{i}^{0} \subseteq B(z, 2 r), i \in \mathbf{Z}$, inequality (4.2) assures that

$$
\int_{\mathbf{R}^{n}} \Phi\left(\left\|\mathbf{M} f^{0}(x)\right\|_{l^{q}}\right) \omega(x) d x \leq C u(z, 2 r) .
$$

We have

$$
\frac{1}{u(z, r)} \int_{B(z, r)} \Phi\left(\left\|\mathbf{M} f^{0}(x)\right\|_{l^{q}}\right) \omega(x) d x \leq C \frac{u(z, 2 r)}{u(z, r)} \leq C
$$

because inequality (3.2) yields $u(z, 2 r)<C u(z, r)$ for some constant $C>0$ independent of $z \in \mathbf{R}^{n}$ and $r>0$. Since $\Phi \in \triangle_{2}$, we obtain

$$
\|\| \mathbf{M} f^{0}\left\|_{l^{q}}\right\|_{M_{\Phi, \omega}^{u}} \leq C
$$

for some $C>0$ independent of $f$. 
As $f_{i}^{j}=\chi_{B_{j+1} \backslash B_{j}} f_{i}$ and $\operatorname{dist}\left(B(z, r), B_{j+1} \backslash B_{j}\right)=\left(2^{j}-1\right) r$, there is a constant $C>0$ such that, for any $j \geq 1$ and $i \in \mathbf{Z}$

$$
\chi_{B(z, r)}(x)\left(\mathrm{M} f_{i}^{j}\right)(x) \leq C 2^{-j n} r^{-n} \chi_{B(z, r)}(x) \int_{B_{j+1}}\left|f_{i}(y)\right| d y .
$$

Since $l^{q}$ is a Banach lattice, we find that

$$
\chi_{B(z, r)}(x)\left\|\left\{\left(\mathbf{M} f_{i}^{j}\right)(x)\right\}_{i \in \mathbf{Z}}\right\|_{l^{q}} \leq C 2^{-j n} r^{-n} \chi_{B(z, r)}(x) \int_{B_{j+1}}\left\|\left\{f_{i}(y)\right\}_{i \in \mathbf{Z}}\right\|_{l^{q}} d y .
$$

In view of the fact that $\omega \in A_{i_{\Phi}}$, by using the openness property of the $A_{i_{\Phi}}$ weight function, there exists a $1<p<i_{\Phi}$ such that $\omega \in A_{p}$. Consequently, the Hölder inequalities assert that

$$
\begin{aligned}
& \int_{B_{j+1}}\left\|\left\{f_{i}(y)\right\}_{i \in \mathbf{Z}}\right\|_{l q} d y \\
& \leq\left(\int_{B_{j+1}}\left\|\left\{f_{i}(y)\right\}_{i \in \mathbf{Z}}\right\|_{l^{q}}^{p} \omega(y) d y\right)^{\frac{1}{p}}\left(\int_{B_{j+1}} \omega^{-\frac{p^{\prime}}{p}}(y) d y\right)^{\frac{1}{p^{\prime}}} \\
& \leq \frac{2^{(j+1) n} r^{n}}{\left(\omega\left(B_{j+1}\right)\right)^{\frac{1}{p}}}\left(\int_{B_{j+1}}\left\|\left\{f_{i}(y)\right\}_{i \in \mathbf{Z}}\right\|_{l^{q}}^{p} \omega(y) d y\right)^{\frac{1}{p}} .
\end{aligned}
$$

Let $\mu$ be the restriction of the weighted measure $\frac{1}{u\left(B_{j+1}\right)} \omega(x) d x$ on $B_{j+1}$. Since $1<p<i_{\Phi}$, $L_{\Phi, \mu}$ is $p$-convex. Therefore, $\left(L_{\Phi, \mu}\right)_{[p]}$ is a Banach function space and the Hölder inequality on $\left(L_{\Phi, \mu}\right)_{[p]}$ is valid. Hence,

$$
\begin{aligned}
\int_{B_{j+1}}\left\|\left\{f_{i}(y)\right\}_{i \in \mathbf{Z}}\right\|_{l^{q}}^{p} \omega(y) d y & \leq u\left(B_{j+1}\right)\|\|\left\{f_{i}\right\}_{i \in \mathbf{Z}}\left\|_{l^{q}}^{p}\right\|_{\left(L_{\Phi, \mu}\right)_{[p]}}\left\|\chi_{B_{j+1}}\right\|_{\left(L_{\Phi, \mu}\right)_{[p]}^{\prime}} \\
& \leq\|\|\left\{f_{i}\right\}_{i \in \mathbf{Z}}\left\|_{l^{q}}\right\|_{\left(\Phi, u, B_{j+1}, \omega\right)}^{p} \frac{\omega\left(B_{j+1}\right)}{\left\|\chi_{B_{j+1}}\right\|_{\left(\Phi, u, B_{j+1}, \omega\right)}^{p}}
\end{aligned}
$$

where we use (2.1) and the fact that $\|\cdot\|_{L_{\Phi, \mu}}$ and $\|\cdot\|_{\left(\Phi, u, B_{j+1}, \omega\right)}$ are identical.

For any $x \in B(z, r)$, Inequalities (4.4)-(4.6) yield

$$
\left\|\left\{\left(\mathbf{M} f_{i}^{j}\right)(x)\right\}_{i \in \mathbf{Z}}\right\|_{l^{q}} \leq C \frac{1}{\left\|\chi_{B_{j+1}}\right\|_{\left(\Phi, u, B_{j+1}, \omega\right)}}\|\|\left\{f_{i}\right\}_{i \in \mathbf{Z}}\left\|_{l q}\right\|_{\left(\Phi, u, B_{j+1}, \omega\right)} .
$$

Applying the norm $\|\cdot\|_{(\Phi, u, B(z, r), \omega)}$ on both sides of the above inequality, we have

$$
\begin{aligned}
& \|\|\left\{\left(\mathbf{M} f_{i}^{j}\right)\right\}_{i \in \mathbf{Z}}\left\|_{l^{q}}\right\|_{(\Phi, u, B(z, r), \omega)} \\
& \leq C \frac{\left\|\chi_{B(z, r)}\right\|_{(\Phi, u, B(z, r), \omega)}}{\left\|\chi_{B_{j+1}}\right\|_{\left(\Phi, u, B_{j+1}, \omega\right)}}\|\|\left\{f_{i}\right\}_{i \in \mathbf{Z}}\left\|_{l q}\right\|_{\left(\Phi, u, B_{j+1}, \omega\right)} \\
& \leq C \frac{\left\|\chi_{B(z, r)}\right\|_{(\Phi, u, B(z, r), \omega)}}{\left\|\chi_{B_{j+1}}\right\|_{\left(\Phi, u, B_{j+1}, \omega\right)}}\|f\|_{M_{\Phi, \omega}^{u}} .
\end{aligned}
$$


Thus, by taking the summation over $j$ on both sides of the above inequality, the vector-valued maximal inequality on weighted Orlicz-Morrey space follows from (3.2).

Whenever, $\Phi(t)=t^{p}, 1<p<\infty$, Theorem 4.2 generalizes the vector-valued maximal inequality on Morrey spaces [29] to weighted Morrey spaces. Moreover, Theorem 4.2 also extends the weighted vector-valued inequality obtained in [1] from Lebesgue spaces to Orlicz spaces and Morrey spaces.

There is another version of vector-valued maximal inequality on Orlicz-Morrey space. It is associated with the Orlicz-Morrey spaces given by the following definition [7, Definition 5.1].

Definition 4.1. Let $v: \mathbf{R}^{n} \times(0, \infty) \rightarrow(0, \infty)$ be a Lebesgue measurable function. A locally integrable function $f$ belongs to $\mathcal{M}_{\Phi}^{v}$ if

$$
\|f\|_{\mathcal{M}_{\Phi}^{v}}=\sup _{x \in \mathbf{R}^{n}, r>0} \frac{1}{v(x, r)}\left\|\chi_{B(x, r)} f\right\|_{L_{\Phi}}<\infty
$$

where $L_{\Phi}$ denotes the Orlicz space associated with the Lebesgue measure on $\mathbf{R}^{n}$.

We write $\mathcal{M}_{p}^{v}$ to denote the above Orlicz-Morrey space associated with $\Phi(t)=t^{p}$, $1 \leq p<\infty$.

Notice that the above definition of $\mathcal{M}_{\Phi}^{v}$ relies only on the fact that $L_{\Phi}$ is a normed linear space, which is independent of the condition that it is generated by modulars. On the other hand, the weighted Orlicz-Morrey space $M_{\Phi, \omega}^{u}$ given in Definition (3.1) is defined via the family of modulars (3.1).

Moreover, the above definition can be generalized to obtain the Morrey space associated with any Banach function space $X$ by replacing the norm $\|\cdot\|_{L_{\Phi}}$ with $\|\cdot\|_{X}$. For instance, in [7], the Morrey space associated with r.-i. Banach function space is introduced and it is further extended to general Banach function spaces in [9].

Whenever $\Phi(t)=t^{p}, 1 \leq p<\infty$ and $v(x, t)=u(x, t)^{\frac{1}{p}}$, we have $M_{p}^{u}=\mathcal{M}_{p}^{v}$.

We state the vector-valued maximal inequality for $\mathcal{M}_{\Phi}^{v}$ in the subsequent theorem.

THEOREM 4.3. Let $1<q<\infty$ and $\Phi \in \triangle_{2} \cap \nabla 2$. If $v$ satisfies

$$
\sum_{j=0}^{\infty} \frac{\left\|\chi_{B(x, r)}\right\|_{L_{\Phi}}}{\left\|\chi_{B\left(x, 2^{j+1} r\right)}\right\|_{L_{\Phi}}} v\left(x, 2^{j+1} r\right) \leq C v(x, r)
$$

for all $x \in \mathbf{R}^{n}$ and $r>0$ for some $C>0$, then

$$
\|\| \mathbf{M} f\left\|_{l^{q}}\right\|_{\mathcal{M}_{\Phi}^{v}} \leq A\|\| f\left\|_{l^{q}}\right\|_{\mathcal{M}_{\Phi}^{v}}
$$

for some $A>0$ independent of $f$.

For the proof of the above result, the reader is referred to [7, Theorem 5.2]. When $\Phi(t)=t^{p}, 1<p<\infty, \omega \equiv 1$ and $v(x, t)=u(x, t)^{\frac{1}{p}},(3.2)$ and (4.7) are identical. 
We present an application of the vector-valued maximal inequalities in weighted OrliczMorrey spaces to the study of function spaces. It follows from a general approach introduced in [7].

We give the definition the weighted Triebel-Lizorkin-Orlicz-Morrey spaces in the following.

Definition 4.2. Let $0<p, q<\infty$. Let $\Phi \in \triangle_{2} \cap \nabla_{2}$ be a Young function, $\omega \in A_{i_{\Phi}}$ and $u$ be an Orlicz-Morrey weight function for $L_{\Phi, \omega}$. The weighted Triebel-Lizorkin-OrliczMorrey space $\dot{F}_{l q,\left(M_{\Phi, \omega}^{u}\right)_{[p]}}^{\alpha}$ consists of those $f \in \mathcal{S}^{\prime}\left(\mathbf{R}^{n}\right) / \mathcal{P}$ satisfying

$$
\|f\|_{\dot{F}_{l q,\left(M_{\Phi, \omega}^{u}[p]\right.}^{\alpha}}^{\alpha}=\|\|\left\{\mid \varphi_{j} * f \|\right\}_{j=-\infty}^{\infty}\left\|_{l q}\right\|_{\left(M_{\Phi, \omega}^{u}\right)_{[p]}}<\infty
$$

where $\varphi_{j}(x)=2^{j n} \varphi\left(2^{j} x\right)$ and $\varphi \in \mathcal{S}\left(\mathbf{R}^{n}\right)$ satisfies

$$
\operatorname{supp} \hat{\varphi} \subseteq\left\{x \in \mathbb{R}^{n}: 1 / 2 \leq|x| \leq 2\right\} \quad \text { and } \quad|\hat{\varphi}(\xi)| \geq C, 3 / 5 \leq|x| \leq 5 / 3
$$

for some $C>0$.

Inhomogeneous weighted Triebel-Lizorkin-Orlicz-Morrey spaces are defined in a similar way. For brevity, we leave the details to the readers.

Using the terminologies given in [7], Theorem 4.2 asserts that the pair $\left(l^{q},\left(M_{\Phi, \omega}^{u}\right)_{[p]}\right)$ is admissible, $0<p, q<\infty$. Thus, [7, Theorem 3.1] assures that the weighted TriebelLizorkin-Orlicz-Morrey spaces is well-defined.

THEOREM 4.4. Let $0<p, q<\infty$. Let $\Phi \in \triangle_{2} \cap \nabla_{2}$ be a Young function, $\omega \in A_{i_{\Phi}}$ and $u$ be an Orlicz-Morrey weight function for $L_{\Phi, \omega}$. The weighted Triebel-Lizorkin-OrliczMorrey space $\dot{F}_{l q,\left(M_{\Phi, \omega}^{u}\right)_{[p]}}^{\alpha}$ is independent of the function $\varphi$ used in Definition 4.2.

The weighted Triebel-Lizorkin-Orlicz-Morrey spaces share those important features of the classical Triebel-Lizorkin spaces such as the boundedness of the $\varphi$ - $\psi$ transforms and the validity of the atomic and molecular decompositions. As they are standard results from the general approach given in [7], for simplicity, we referred the readers to [7] and [8] for details.

ACKNOWLEDGMENT. The author would like to thank the referees for their valuable suggestions and careful reading which greatly improve the presentation and the context of this paper.

\section{References}

[ 1 ] Andersen, K. and John, R., Weighted inequalities for vector-valued maximal functions and singular integrals, Studia Math. 69 (1980), 19-31.

[2] Bennett, C. and Sharpley, R., Interpolation of Operators, Academic Press, 1988.

[ 3 ] Cruz-Uribe, D., Martell, J. and PÉrez, C., Weights, Extrapolation and the Theory of Rubio de Francia, Operator Theory: Advance and Applications, Volume 215, Birkhäuser Basel (2011). 
[4] Curbera, G., García-Cuerva, J., Martell, J. and Pérez, C., Extrapolation with weights, rearrangement-invariant function spaces, modular inequalities and applications to singular integrals, Adv. Math. 203 (2006), 256-318.

[ 5 ] Fefferman, C. and Stein, E., Some maximal inequalities, Amer. J. Math. 93 (1971), 107-115.

[6] Grafakos, L., Modern Fourier Analysis, second edition, Springer, 2009.

[ 7 ] Ho, K.-P., Littlewood-Paley spaces, Math. Scand. 108 (2011), 77-102.

[ 8 ] Ho, K.-P., Wavelet bases in Littlewood-Paley spaces, East J. Approx. 17 (2011), 333-345.

[9] Ho, K.-P., Vector-valued singular integral operators on Morrey type spaces and variable Triebel-LizorkinMorrey spaces, Ann. Acad. Sci. Fenn. Math. 37 (2012), 375-406.

[10] Ho, K.-P., The fractional integral operators on Morrey spaces with variable exponent on unbounded domains, Math. Inequal. Appl. 16 (2013), 363-373.

[11] Ho, K.-P., Atomic decompositions of weighted Hardy-Morrey spaces, Hokkaido Math. J. 42 (2013), $131-157$.

[12] Kokilashvilli, J. and Krbec, M., Weighted Inequalities in Lorentz and Orlicz spaces, World Scientific (1991).

[13] KuRTZ, D., Littlewood-Paley and multiplier theorems on weighted $L^{p}$ spaces, Trans. Amer. Math. Soc. 259 (1980), 235-254.

[14] Lindenstrauss, J. and Tzafriri, L., Classical Banach Spaces I and II, Springer, New York, (1996).

[15] Mazzucato, A., Decomposition of Besov-Morrey spaces. Harmonic analysis at Mount Holyoke (South Hadley, MA, 2001), Contemp. Math. 320 Amer. Math. Soc. (2003), 279-294.

[16] Morrey, C., On the solutions of quasi-linear elliptic partial differential equations, Trans. Amer. Math. Soc. 43 (1938), 126-166.

[17] MusielaK, J., Orlicz Spaces and Modular Spaces, Springer (1983).

[18] NAKAI, E., Hardy-Littlewood maximal operator, singular integral operators and the Riesz potentials on generalized Morrey spaces, Math. Nachr. 166 (1994), 95-104.

[19] NaKaI, E., Recent topics of fractional integrals, Sugaku Expositions 20 (2007), 215-235.

[20] NAKAI, E., Orlicz-Morrey spaces and the Hardy-Littlewood maximal function, Studia Math. 188 (2008), 193-221.

[21] NaKano, H., Modular Semi-Orderd Linear Spaces, Maruzen Co. Ltd. (1950).

[22] Okada, S., Ricker, W. and SÁnchez PÉrez, E., Optimal Domain and Integral Extension of Operators, Birkhäuser Basel (2008).

[23] Pietsch, A., History of Banach spaces and linear operators, Birkhäuser, Boston, 2007.

[24] Rao, M. and Ren, Z., Theory of Orlicz Spaces, Dekker, New York, 1991.

[25] Sawano, Y. and Tanaka, H., Morrey spaces for non-doubling measures, Acta Math. Sin. (Engl. Ser.) 21 (2005), no. 6, 1535-1544.

[26] Sawano, Y. and Tanaka, H., Decompositions of Besov-Morrey spaces and Triebel-Lizorkin-Morrey spaces, Math. Z. 257 (2007), 871-905.

[27] Sawano, Y., Sobukawa, T. and Tanaka, H., Limiting case of the boundedness of fractional integral operators on nonhomogeneous space, J. Inequal. Appl. (2006), Art. ID 92470, 16 pp.

[28] Sawano, Y., Sugano, T. and Tanaka, H., Orlicz-Morrey spaces and fractional operators, Potential Anal. 36 (2012), no. 4, 517-556.

[29] TANG, L. and XU, J., Some properties of Morrey type Besov-Triebel spaces, Math. Nachr. 278 (2005), 904917.

[30] WAnG, H., Decomposition for Morrey type Besov-Triebel spaces, Math. Nachr. 282 (2009), 774-787. 
Present Address:

DEPARTMENT OF MATHEMATICS AND INFORMATION TECHNOLOGY,

The Hong Kong Institute of Education,

10, Lo Ping Road, Tai Po, Hong Kong, China.

e-mail: vkpho@ied.edu.hk 